\title{
marges Marges
}

revue d'art contemporain Revue d'art contemporain

$17 \mid 2013$

Remake, reprise, répétition

\section{Replace or remake ? Considérations sur la notion de « remake » en conservation-restauration}

Replace or remake? Remarks on the Notion of "remake" in the CuratingRestoration Field

Marie-Hélène Breuil

\section{(2) OpenEdition}

\section{Journals}

Édition électronique

URL : http://journals.openedition.org/marges/169

DOI : 10.4000/marges.169

ISSN : 2416-8742

\section{Éditeur}

Presses universitaires de Vincennes

Édition imprimée

Date de publication : 1 novembre 2013

Pagination : 115-122

ISBN : 978-2-84292-394-5

ISSN : $1767-7114$

\section{Référence électronique}

Marie-Hélène Breuil, « Replace or remake ? Considérations sur la notion de « remake » en

conservation-restauration », Marges [En ligne], 17| 2013, mis en ligne le 01 novembre 2014, consulté le 24 avril 2019. URL : http://journals.openedition.org/marges/169; DOI : 10.4000/marges.169 


\section{Replace or remake?}

Considérations sur la notion

de «remake» en

conservation-restauration

/1 Cet article s'appuie sur des recherches en cours menées dans le cadre de l'Équipe de recherche sur la conservation-restauration des œuvres sculptées, ER[cr]OS, de l'École supérieure des beaux-arts TALM Site de Tours en 2012 et 2013. Nos remerciements à Anne Cadenet, chargée du Service Collection, Documentation, Archives, CAPC-Musée d'art contemporain, Bordeaux et à Christophe Domino, critique d'art et professeur à l'Esba TALM Site du Mans.

/ 2 « Le ConservateurRestaurateur est un professionnel qui a la formation, la connaissance, les aptitudes, l'expérience et les facultés de compréhension pour agir dans le but de préserver les biens culturels pour le futur ", Code de déontologie, ECCO
Depuis quelques années, la question de la préservation des œuvres d'art contemporain se pose de façon accrue et les recherches à ce sujet se multiplient, portant aussi bien sur les matériaux constitutifs des œuvres et sur les matériaux de restauration que sur la déontologie de la profession/1. Les conservateurs-restaurateurs/2 sont aujourd'hui confrontés à de nouveaux défis ainsi qu'au paradoxe d'une muséification qui implique de préserver ce qui n'est pas toujours conservable. Au sein du département Conservation-restauration des œuvres sculptées de l'École supérieure des beaux-arts TALM Site de Tours nous avons travaillé à plusieurs reprises sur des œuvres dont la précarité matérielle impliquait que tout ou partie soit remplacé ou refait en vue de leur exposition dans le maintien de leur fonction et de leur signification. La restauration de Sans titre de Fabrice Hyber, une œuvre de 1989 conservée au Frac des Pays de la Loire, nous a conduit en 1999 à proposer de conserver en réserve l'élément central de l'œuvre - une figure en pied autoportrait et autographe de l'artiste immergée dans un aquarium - et à réaliser une copie d'exposition de cette même figure. Pierre Mouchard, l'étudiant en charge de cette restauration, avait alors très judicieusement nommé cette copie une « réplique fonctionnelle », précisant: « Nous dissocierons sur le plan physique la matière en tant que vecteur de l'histoire et la matière en tant que vecteur renouvelé de l'esthétique et du fonctionnement. [...] Les responsables du Frac auront de leur côté à réfléchir au moyen d’indiquer la nature réelle du personnage restitué par la rédaction d'un cartel d'exposition; celui-ci est un des dispositifs les plus à même de prévenir les interprétations erronées 
(European Confederation of Conservator-Restorers' Organisations), 2002. En ligne: [http://www. ecco-eu.org/about-e.c.c.o./ professional-guidelines. html]

/3 Pierre Mouchard, Étude, restauration et restitution d'une œuvre de Fabrice Hybert, Sans titre, mémoire de fin d'études en Conservationrestauration des œuvres sculptées, École supérieure des beaux-arts de Tours, 1999, p. 59 et 60 .

/4 Voir à ce sujet Restauration et nonrestauration en art contemporain 1, actes des journées d'étude du cursus conservationrestauration des œuvres sculptées de l'École supérieure des beaux-arts de Tours, 14 février et 6 avril 2007, Tours, ARSET, 2008, notamment: Paul-Hervé Parsy, "Y a-t-il un cas Fountain ", p. 103-113; Guylène Mary, "La restauration de Fountain », p. 115-125.

/5 « Terminology for further expansion ", Tate Papers, $\mathrm{n}^{\circ} 8$, Automne 2007. Special issue on Replication for the workshop « Inherent Vice: The Replica and its Implications in Modern Sculpture » held at the Tate Modern, 18-19 October 2007. En ligne: [http://www.tate.org.uk/ research/publications/ tate-papers/issue-08]

/6 Une autre distinction à retenir, que nous n'abordons pas dans cet article, est de l'objet, interprétations susceptibles d'en permettre l'assimilation à un faux/3. ». En 2007 nous sommes revenus sur cette question dans le cadre de la journée d'étude Répliques et restitutions... autour de Marcel Duchamp qui nous a permis de questionner la pratique et la déontologie du conservateur-restaurateur confronté à la perte ou à la ruine d'œuvres ainsi que les réponses et les paradoxes des artistes, l'édition (le remake?) de ses ready-mades par Marcel Duchamp par exemple/4.

Le remplacement et le fait de refaire ne sont acceptés qu'avec des réserves dans la déontologie-on parlealors de « reconstitution »-, mais il se trouve que les débats sur la conservation des œuvres contemporaines ont vu ces dernières années l'introduction du terme de remake. Il apparaît dans le lexique élaboré en 2007 par la Tate Modern à l'occasion du workshop Inherent Vice: The Replica and its Implications in Modern Sculpture: "Remake - item fait à l'origine par l'artiste, puis refait (par l'artiste, ou par quelqu'un sanctionné par l'artiste) en utilisant des matériaux nouveaux/5. ". La dégradation irréversible des matériaux constitutifs de plusieurs sculptures de Naum Gabo conservées à la Tate est à l'origine de ce workshop: l'instabilité de l'acétate de cellulose considéré alors comme un matériau stable et choisi par l'artiste pour sa nouveauté et sa modernité, conduit à la disparition de ces œuvres et pose la question de leur reproduction. L'introduction d'un vocabulaire nouveau dans le domaine de la conservation est en adéquation avec le changement des pratiques et des positions artistiques opéré dans l'art contemporain et la nécessité qui s'en est suivie d'adapter la déontologie de la profession. Le glissement syntaxique du verbe (to re-make) au substantif est suivi d'un glissement linguistique alors même que les symposiums internationaux sur la question de la conservation des œuvres contemporaines sont de plus en plus nombreux. Mais ce terme n'est pas sans poser de problèmes, non seulement du fait de ses connotations cinématographiques qui impliquent une interprétation sinon une sur-interprétation d'un film à succès, mais surtout parce que le statut de l'œuvre " refaite » ne semble pas toujours très clair, oscillant entre la copie d'exposition, la duplication, la reproduction ou reconstruction considérée ou non par l'artiste au même titre que l'original. Les confusions qui en résultent proviennent souvent du manque de documentation sur la réplique (entendue ici au sens large replica). En effet, la définition et la fonction du remake dépendent du statut même de son auteur, celui qui refait, et du contexte de re-production. La sanction de l'artiste viendra appuyer le statut du remake, lui conférant par sa reconnaissance une authenticité certaine en tant que tel. Aussi nous nous proposons de ne considérer ici que les remakes réalisés par des 
conservateurs-restaurateurs/6 et de laisser de côté ceux réalisés par les artistes qui posent notamment les questions de l'authenticité au regard de l'original, de la série et de l'édition.

L'occurrence des termes dans les textes fondamentaux de la conservation-restauration révèle une sorte de flottement dans leur définition même: comme les images les mots sont sujets à interprétation. La Théorie de la restauration de Cesare Brandi est incontournable pour la discipline. Publié en 1963, mais traduit tardivement en français, ce texte pose les principes et les règles d'une discipline en constitution dont les catégories sont entièrement calquées sur celles des beaux-arts, alors même que celles-ci sont remises en question. Dans sa définition, la conservation-restauration entend alors se distinguer du travail artistique (dans le sens où le restaurateur ne produit pas d'œuvre d'art) et de l'artisanat (dans le sens ou le savoir-faire est théorisé et objectivé) en s'appuyant sur un principe déontologique : ne pas commettre de faux artistique ou de faux historique/7. Brandi écarte toute possibilité de refaire tout ou partie d'une œuvre: «Il ne suffit pas, même avec la documentation la plus vaste et la plus minutieuse, de savoir ce qu'était l'œuvre avant de devenir une ruine. La reconstruction, la restitution, la copie ne peuvent même pas être abordées à propos de la restauration; elles sortent naturellement du sujet pour entrer uniquement dans le domaine de la légitimité ou de l'illégitimité de la reproduction "à froid » des procédés aboutissant à la formulation de l'œuvre d'art/8. ». Pour Brandi « la copie est un faux à la fois historique et esthétique; elle peut avoir uniquement une justification didactique et commémorative [...]/9 ». Cette idée est reprise par Ségolène Bergeon-Langle qui admet cependant la possibilité, en toute légitimité, de la reconstitution: "Reconstitution - Une œuvre ou les parties disparues d'une œuvre peuvent être remises sous les yeux de deux manières: virtuelle ou réelle. Dans le premier cas on parlera de restitution. La reconstitution appartient au contraire au monde réel. Quelle que soit la précision des documents sur lesquels elle se fonde, la reconstitution n’a jamais pour résultat la résurrection de l'original: ce serait un contre-sens logique. Elle aboutit à la production d'un substitut. L'opération n'a rien d'illégitime si elle se donne pour ce qu'elle est. En revanche la reconstitution qui ne s'avoue pas, ou se masque sous le mot de restitution, est une falsification/10. ". Dans cette définition la reconstitution est très clairement un remake, bien qu'il ne soit pas fait mention de la sanction de l'artiste. Son statut est celui d'un substitut qui remplace en "remettant sous les yeux ». II s'agit de donner à voir ce qui est perdu ou n'est plus présentable. En ce sens, la « reconstitution » peut être assimilée à un fac-similé, au sens où la distinction entre le remake et les œuvres issues d'une matrice: la reproductibilité technique à partir d'un négatif ou d'un master pour la photographie, le cinéma ou la vidéo; les procédés de reproduction en sculpture à partir d'empreinte, le moulage notamment.

/7 «[...] le conservateurrestaurateur ne crée pas d'objets culturels nouveaux. Reconstruire physiquement ce qui n'existe plus ou ne peut être préservé est du domaine de l'artisanat ou des professions artistiques » lit-on dans « Le conservateurrestaurateur: une définition de la profession », 4.1, Comité pour la conservation, Conseil international des musées (ICOM), Copenhague, septembre 1984. [http:// www.ffcr.fr/ffcr_ download/referentiels/ Icom.pdf ]

/8 Cesare Brandi, Théorie de la restauration, Paris, Centre des Monuments nationaux/Monum, Editions du patrimoine, 2001, p. 54.

/9 ibid., p. 74. La question du faux revient souvent dans la théorie de Brandi, il consacre d'ailleurs un appendice à ce sujet: "Falsification », p. 91-95.

/10 Ségolène BergeonLangle, Georges Brunel, "Original et reproduction, questions de vocabulaire ", Coré, $\mathrm{n}^{\circ} 22$, juillet 2009, p. 6. 
/11 Point 4.7 du Code de déontologie pour les musées, ICOM, 2006. [http://archives.icom. museum/code2006_fr. pdf]

/12 Nathalie Leleu, " "Mettre le regard sous le contrôle du toucher" Répliques, copies et reconstitutions au $\mathrm{xx}^{\mathrm{e}}$ siècle: les tentations de l'historien de l'art", Les Cahiers du Musée national d'art moderne, $n^{\circ} 93$, automne 2005 , p. 85 .

/13 Cette confusion se rencontre nous semble-t-il dans l'article de Kerstin Luber et Barbara Sommermeyer, « Remaking Artworks: Realized Concept versus Unique Artworks », dans Tatja Scholte et GlennWharton (sld.), Inside Installations, Theory and Practice in the Care of Complex Artworks, Amsterdam, Amsterdam University Press, 2011, p. 235-248. l'entend le Code de déontologie pour les musées de l'ICOM à l'entrée "Reproductions »: "Les musées doivent respecter l'intégrité des originaux lorsqu'ils exécutent des répliques, des reproductions ou des copies d'articles de la collection. Toutes ces copies doivent être en permanence signalées comme fac-similés/11. ».

Citons encore: "Il faut entendre par réplique, copie et reconstitution, la restitution d'une œuvre d'art dans son état originel. Cette reproduction ne résulte pas d'une matrice, et elle n'est pas non plus une conséquence du processus créatif conçu par l'auteur du prototype: elle restitue une forme plastique autonome. C'est ainsi que la réplique, la copie et la reconstitution ne sauraient relever de l'édition et du multiple, qui dérivent d'une convention ou d'un concept a priori/12. ». Toujours selon Nathalie Leleu, la réplique est « la répétition (avec ou sans modification) d'un original, exécutée par l'artiste ou sous son contrôle ", définition proche, bien que plus large, de celle du terme remake que l'on trouve dans le lexique de la Tate Modern. La pratique du remake en conservation-restauration croise les pratiques de réitération, ré-instauration ou encore d'actualisation qui se rencontrent dans les discours et propositions des artistes ce qui engendre une certaine confusion notamment à l'encontre d'œuvres assimilées à l'art conceptuel pour lesquelles l'idée, la conception (et j'emploie précisément ces termes et non celui de « concept »), intègre la délégation de réalisation/13. Le restaurateur est pris dans un chassé-croisé alimenté par l'idée généralement acceptée que l'art conceptuel est immatériel. Or, à l'examen, il s'avère que les œuvres conceptuelles ont une forte matérialité et que les artistes sont très soucieux du soin apporté à leur réalisation. C'est donc autant la temporalité de leur réalisation et la conscience que l'œuvre n'existe matériellement que le temps de son exposition qui les caractérisent que la délégation de réalisation qui sont comprises dans leur définition. En ce sens il nous paraît essentiel de distinguer l'actualisation du remake: ainsi les wall drawings de Sol LeWitt réalisées pour l'exposition du Centre Pompidou Metz sont de notre point de vue des actualisations; de même qu'une définition/méthode de Claude Rutault sera actualisée pour chaque exposition, repeinte et donc refaite, sans pour autant qu'elle ne soit un remake. Le remake est destiné à être conservé contrairement à ces œuvres spécifiquement conçues pour être produites à la demande et donc produites à nouveau et qui, de fait, excluent la conservation.

Mais la problématique n'est pas que de vocabulaire, elle concerne les éléments matériels disparus d'une œuvre ainsi que les éléments dont la dégradation modifie la signification ou ceux devenus obsolètes et nécessitant un remplacement afin d'assurer une continuité 
de fonctionnement et de compréhension de l'œuvre: duplicata des tirages photos, remplacement des néons, migration de contenu pour les œuvres audiovisuelles, etc. Dans tous les cas le remake se distingue de l'original, si ce n'est dans le matériau, les dimensions, la mise en œuvre, au moins dans le fait qu'il résulte d'une volonté de refaire une œuvre dans un contexte donné, évidemment différent du contexte d'origine.

La plupart des remakes réalisés par des restaurateurs ont une fonction documentaire et/ou éducative : il s'agit de donner à voir au public, ou encore de comprendre comment l'œuvre est faite en la refaisant. Il arrive que, du fait de la trop grande dégradation de l'original et de sa possible perte imminente, ils prennent une valeur différente de leur fonction et deviennent des répliques ou copies d'exposition se substituant à l'original ce qui nécessite de les documenter afin de définir leur statut. Ainsi le marquage des remakes en tant que tel s'impose afin d'éviter les falsifications ou les utilisations inappropriées. Le problème majeur n'est pas la réalisation du remake mais bien la question de son utilisation et des problèmes juridiques et économiques que ces objets soulèvent.

Dans le cadre du projet Replace or remake/14 engagé en 2012, nous avons été sollicité par le CAPC-Musée d'art contemporain à Bordeaux afin d'étudier une œuvre de Richard Long réalisée en 1990 à l'occasion de la réouverture du musée, White Rock Line, une ligne de 40 mètres de longueur et 18 tonnes de pierre installée sur l'une des terrasses du CAPC-Musée d'Art contemporain à Bordeaux/15. En 1990, et jusqu'à une période récente, White Rock Line faisait pendant à Ligne d'ardoise Bordeaux, une œuvre commandée à l'artiste par le FRAC Aquitaine en 1984 et conçue pour une première installation au CAPC. La ligne de pierre blanche s'opposait alors à la ligne d'ardoise, noire. La pierre calcaire, blanche comme l'indique le titre, est aujourd'hui devenue très grise, les moellons s'altèrent et certains se sont brisés. L'étude que nous menons porte sur le matériau et les conditions de conservation, ainsi que sur l'histoire de l'œuvre en vue de sa préservation conformément aux indications portées sur le certificat. La description et les dessins qu'il comporte nous renseignent sur sa réalisation et son installation ainsi que sur son maintien en l'état: "La sculpture comprend entre 1 et 3 couches de pierres, couvrant solidement le sol. Les pierres du bord doivent être particulièrement stables, et la couche supérieure de pierres doit être placée au hasard et de façon chaotique. Il ne doit pas y avoir de pierres en équilibre. La sculpture est un peu plus épaisse en son centre, et il doit y avoir une apparence de densité tout le long. Les angles sont droits. Toute pierre qui tomberait hors de l'œuvre doit
/14 Replace or remake est le titre du projet de recherche mené par ER[cr] OS, Équipe de recherche sur la conservationrestauration des œuvres sculptées, École supérieure des beaux-arts TALM Site de Tours en 2012 et 2013.

/15 Une image de cette œuvre est visible à l'adresse suivante : [http://www.capcbordeaux.fr/richardlong...] 
/16 Certificat manuscrit, archives du CAPC-Musée d'art contemporain, Bordeaux. Traduction de l'auteur.

Le certificat a pour fonction première d'authentifier l'œuvre, il accompagne l'œuvre mais ne peut être reproduit ou exposé.

/17 Richard Long, entretien avec Christophe Domino, archives Christophe Domino. A Pipe Maker's Wood Line est une longue ligne réalisée en 1997 à partir des résidus de blocs de bruyère utilisés par un artisan corse lors de la fabrication de pipes. L'œuvre refaite en 2007 à la demande du FRAC Corse est dorénavant datée 1997-2007.

/18 Avec Marion Carel, Mathilde Champdavoine, Augustin Laforêt, Camille Leconte, Morgane Poirier, Eglantine Ruaudel, Celia Santi, Olivier Steib et Muzhi Wang, étudiants. Cette étude donnera lieu à une publication spécifique.

\section{/19 Un cercle en} Bretagne, 1986, Kerguéhennec (France); Sea Lava Circles, 1988, Chinati Foundation, Marfa (Etats-Unis) ; Tagliamento River Stone Ring, 1996, Verzegnis, Udine (Italie) ; Pontevedra Line, 1999, Pontevedra (Espagne); Energy Axis, 1999, Braga (Portugal); Forte di Vinadio Circle, 2001, Vinadio (Italie); Tame Buzzard Line, 2001, East Winterslow (Angleterre); Macduff Circle, 2002, Edinburgh (Ecosse); être replacée dans la ligne. Ce n'est pas une sculpture sur laquelle on marche/16. ». Nous apprenons également que White Rock Line « doit être maintenue aussi propre et blanche que possible " mais sans indication de méthode. Cette information donne une orientation certaine à la conservation de l'œuvre dans un état conforme à son titre et à son origine : une ligne de pierre blanche. Deux options sont donc envisagées: conserver les éléments matériels et les nettoyer, puis les réaligner conformément aux indications du certificat, ce qui implique la mise en place d'un important chantier du fait des dimensions de la pièce; ou refaire l'œuvre, solution qui, juridiquement, ne peut être envisagée qu'en concertation avec l'artiste, dans l'éventualité où le musée ferait le choix d'un remake. Au sujet d'une autre de ses œuvres, refaite avec la collaboration de Christophe Domino à la suite de sa destruction, A Pipe Maker's Wood Line, Richard Long commente: «C'était une très bonne idée que de me donner l'opportunité de la refaire/17 » et il insiste sur son attachement au matériau et à son histoire. Dans le cas de White Rock Line, il s'agit également d'estimer la faisabilité et le coût de l'une ou l'autre des opérations, le remake constituant vraisemblablement dans ce cas-là une opération moins coûteuse pour l'institution qu'un traitement de conservationrestauration.

L'étude technico-scientifique est menée dans le cadre des enseignements du département Conservation-restauration des œuvres sculptées, sous la direction de Marie-Emmanuelle Meyohas/18, à partir d'un échantillonnage d'une dizaine de pierre calcaire prélevées sur l'œuvre et d'autant en provenance directe de la carrière de Malville (située sur la commune de La Tour Blanche en Dordogne) qui vient d'ouvrir une faille de même densité que la pierre achetée en 1990 par le CAPC pour la production de l'œuvre. Elle porte sur l'identification et l'étude des altérations - les pierres étudiées présentent plusieurs types d'attaques de nature biologique: des champignons, des algues et des lichens - et sur la mise au point d'un protocole de nettoyage applicable à grande échelle (bien que long et fastidieux à mettre en œuvre, chaque pierre devant être traitée sur toutes ses faces) ainsi que d'un protocole préventif applicable aux pierres nettoyées comme aux pierres «neuves ».

L'étude historique et contextuelle, complément indispensable à la compréhension de l'œuvre, s'étend à une douzaine de pièces de Richard Long conçues pour l'extérieur, notamment en Italie, Espagne et Grande-Bretagne et installées de façon permanente/19. Elle implique de considérer les intentions de l'artiste dans ce type de production, le statut des œuvres et du certificat d'authenticité qui les accompagne, la place et le devenir de ces œuvres «permanentes/20 » en 
plein air dans le corpus de l'artiste, ainsi que le processus décisionnel du musée. Il est d'ailleurs intéressant de noter que Richard Long se définit comme un artiste du paysage (" a landscape artist/21 ) plutôt que du Land Art. Au-delà des seuls aspects de conservationrestauration, cette proposition permet de questionner les pratiques de remake du point de vue de l'institution, en vue de la présentation et de l'exposition des œuvres, de réfléchir aux conditions d'installation et de réinstallation, d'exposition et de ré-exposition tout en considérant les possibilités de changement (modifications, transformations, remplacement, reconstitution...) de l'œuvre selon son contexte d'exposition, dans le temps et dans l'espace.

À ce jour le peu de réponses au questionnaire que nous avons envoyé aux responsables des différents sites révèle surtout le manque de documentation et la perte d'information sur les conditions même de la production de l'œuvre et ses aspects matériels: la provenance de la pierre par exemple n'est pas toujours connue et il n'est pas certain que toutes ces œuvres soient toujours en place/22. Des informations nous ont cependant été communiquées sur Un cercle en Bretagne/23, réalisé par Richard Long en 1986 au Domaine de Kerguéhennec à Bignan. Le déplacement et la réfection de l'œuvre ont été menés selon les indications transmises par Richard Long: de nouvelles pierres, les mêmes ou plus grosses, ce qui pourrait être mieux pour des raisons pratiques et esthétiques - "look better » indique-t-il - sont placées sur les vieilles pierres - « old (rotten) » précise-t-il dans sa correspondance à Christophe Domino/24, dessin à l'appui. Cet exemple est intéressant à plusieurs titres: non seulement l'artiste n'hésite pas à déléguer l'installation en transmettant des informations où le texte et le dessin se répondent, comme c'est le cas sur les certificats; mais encore les informations sont à la fois précises et générales laissant une marge non pas d'interprétation mais de différenciation dans le détail d'une installation à l'autre; enfin on y comprend que l'empilement et le recouvrement des couches sont importants, procédant par strate, les matériaux nouveaux venant recouvrir les plus anciens comme des couches géologiques, ce qui s'inscrit tout à fait dans la logique du travail de Richard Long.

Revenant sur White Rock Line nous remarquons que le certificat précise: «La sculpture comprend entre 1-3 couches de pierres, couvrant solidement le sol. ». Cette insistance sur le nombre de couches et le fait qu'aujourd'hui, même si l'œuvre est toujours comme l'indique le même certificat « un peu plus épaisse en son centre ", il semble y avoir plutôt deux couches que trois, laisse supposer que la solution du recouvrement des «vieilles pierres » par de nouvelles pourrait être une réponse apportée par l'artiste à la réfection de cette œuvre
Full Moon Circle, 2004, Houghton Hall, Norfolk (Angleterre); Ascott Circle, 2004, Wing (Angleterre); dans le même matériau, une pierre calcaire, et de la même année, bien qu'installée en intérieur: Rochechouart Line, 1990, Musée de Rochechouart (France); et dans un autre matériau, mais en rapport direct avec l'œuvre du CAPC: Ligne d'ardoise, Bordeaux, 1984, FRAC Aquitaine. Cette étude est en cours de réalisation.

/20 L'ensemble de ces $œ u v r e s$ est qualifié de " permanently on view » sur le site Internet:

[http://www.

therichardlongnewsletter. org]

/21 Ce qu'il rappelle dans l'entretien déjà cité avec Christophe Domino.

/22 Parce que ces pièces sont « contemporaines » et l'artiste vivant il semblerait qu'il n'ait pas toujours été jugé utile de les documenter de façon pérenne. Ceci se constate assez régulièrement dans l'étude matérielle des œuvres contemporaines pour lesquelles une trop grande confiance est faite à la transmission de l'information de personne à personne.

/23 Par Christophe Domino qui a également supervisé ce « remake ».

/24 Correspondance de Richard Long avec Christophe Domino, non datée, archives Christophe Domino. 
/ 25 Courrier de Richard Long à Hervé Legros, 26 janvier 2004, indication aimablement transmise par le FRAC Aquitaine.

/ 26 Ideally, this work is permanently placed.

par adjonction de matériaux plutôt que par remplacement. Cette hypothèse ne résout cependant que très partiellement la question de la couleur des pierres et de la ligne, la couche de "fondation" restant alors grise. White Rock Line serait alors une œuvre refaite par ajout et non par remplacement, ce qui écarte la question de la conservation des restes mais n'écarte pas celle de l'entretien régulier de l'œuvre.

Dans un autre cas, au voisinage de White Rock Line puisqu'il s'agit de Ligne d'ardoise Bordeaux du FRAC Aquitaine, Richard Long a conseillé de remplacer les éléments endommagés (brisés et/ou rayés) à la suite de l'exposition de l'œuvre en 2003 au Palais des Papes en Avignon, par des éléments « identiques » de même provenance - la carrière de Delabole en Cornouailles - et de les mélanger aux plaques d'origine/25. Mais il est vrai que, ici, la question de la couleur n'intervenait pas.

Peut-être nous faudrait-il considérer ces œuvres comme des monuments plutôt que comme des pièces de musées: à l'instar des édifices elles nécessitent un entretien et le cas échéant le remplacement des éléments endommagés où l'ajout d'éléments nouveaux afin de pouvoir maintenir leur fonction et leur usage au regard de leur valeur culturelle. Ce sont des œuvres construites mais non architecturées, assemblées sans que rien ne les fixe. De ce fait leur permanence dans le temps et dans l'espace paraît aléatoire. Richard Long conclut le certificat de White Rock Line - et apparemment d'un certain nombre, voire de la totalité, des certificats de ses pièces en extérieur - par la phrase suivante: «Idéalement, cette œuvre est installée de façon permanente/26 ». La pérennité de l'œuvre se trouve donc relativisée au mieux de ce qui est possible ou de ce qu'il lui est possible d'envisager sans donc écarter son éventuelle précarité. White Rock Line est composée de 18 tonnes de pierres et se déploie sur 40 mètres en une ligne au sol qui guide le regard et accompagne notre flânerie sur les terrasses du musée. À l'issue de cette étude, il appartiendra à l'artiste et au musée de se prononcer sur sa réfection.

\section{Marie-Hélène Breuil}

